\title{
Estatinas na doença renal crônica
}

\section{Statins in chronic renal disease}

Soraia R. C. Ferreira, Aloísio M. Rocha, José Francisco K. Saraiva.

Disciplinas de Cardiologia e Nefrologia, Faculdade de Medicina - PUC de Campinas.

Hipertrigliceridemia e o HDL baixo são aspectos comuns em pacientes com insuficiência renal crônica. A mortalidade cardiovascular está substancialmente aumentada na presença de doença renal crônica (10-20 vezes maior). Existem evidências de estudos clínicos com estatinas sugerindo uma ação protetora dessas drogas na progressão da doença renal. Além disso, pacientes póstransplante renal recebendo fluvastatina, experimentaram redução na incidência de infartos não fatais e de mortalidade cardíaca. Entretanto, um estudo recente com atorvastatina não demonstrou reduções na morbimortalidade cardiovascular entre pacientes diabéticos em hemodiálise. Estudos em andamento definirão o preciso papel das estatinas neste grupo especial de pacientes.

\section{Palavras-chave}

Estatina, doença renal crônica, dislipidemia
Hypertriglyceridemia and low HDL-c are common features in patients with chronic renal failure. Cardiovascular mortality is substantially increased in the presence of chronic renal disease (10-20 times higher). There is evidence from clinical trials with statins suggesting their protective role in the progression of renal disease. In addition, reduced rates of non-fatal myocardial infarction and cardiac mortality were seen after renal transplant in patients receiving fluvastatin. However, a recent study with atorvastatin failed to demonstrate reduction in cardiovascular morbidity and mortality among diabetic patients on hemodyalisis therapy. Ongoing trials will define the precise role of statins in this subset of patients.

\section{INTRODUÇÃo}

Anormalidades no metabolismo lipídico são comuns em pacientes com doença renal crônica (DRC) em tratamento conservador, em diálise ou após o transplante renal. Estes pacientes apresentam um perfil lipídico tipicamente trombogênico, com predomínio de elevação dos níveis de triglicérides (TG) e redução do $\mathrm{HDL}$-colesterol (HDL-c). A elevação dos TG é explicada pela redução da sua remoção devido à redução da atividade da lipase lipoprotéica e da lipase triglicerídea hepática. Os níveis do colesterol total (CT) podem ser normais, talvez em parte devido à presença da desnutrição. Estas alterações podem ser observadas em fases iniciais do declínio da função renal (taxa de filtração glomerular $<60 \mathrm{ml} / \mathrm{min})$. Os níveis de VLDL e IDLcolesterol, mas não necessariamente de LDL-c, estão elevados. A composição do LDL é anormal, apresentando aumento no seu conteúdo de TG. Ocorre um acúmulo dos remanescentes de lipoproteínas no plasma dos pacientes com DRC, que são partículas altamente aterogênicas ${ }^{1,2}$.

A mortalidade por doença cardiovascular (DCV) é maior em pacientes com DRC, chegando a ser 10 a 20 vezes superior ao se comparar à população geral, constituindo-se a principal causa de óbito destes pacientes em tratamento conservador, em diálise e após o transplante renal ${ }^{3}$.

O risco excessivo da DCV na DRC é explicado em parte pela elevada prevalência dos fatores de risco cardiovasculares

\section{KEY WORDS}

Statins, end stage renal disease, dyslipidemia

ditos "tradicionais" e também pela presença dos fatores de risco "não tradicionais". Estes incluem anormalidades hemodinâmicas e metabólicas decorrentes da disfunção renal como, por exemplo, a anemia, os distúrbios do metabolismo do cálcio e fósforo, a hipervolemia, aumento da lipoproteína (a), hipoalbuminemia, elevação do paratormônio, acidose, hiperhomocisteinemia, desnutrição, inflamação, estresse oxidativo, proteinúria, fatores trombogênicos, atividade do sistema da renina-angiotensina e toxinas urêmicas ${ }^{4}$.

Levin et al ${ }^{5}$ relatam de forma elegante o paralelismo entre a progressão da doença cardíaca e o declínio da função renal. As duas doenças progridem simultaneamente e têm inúmeros fatores de risco em comum (figura 1), como por exemplo, hipertensão arterial sistêmica (HAS), diabete melito (DM) e dislipidemia. Além disso, existem alguns fatores específicos de cada doença que podem exercer efeito sobre a outra, como por exemplo, as toxinas urêmicas que sabidamente podem afetar a função miocárdica.

É possível que os mesmos fatores que afetam a função cardíaca acelerem o declínio da função renal por ativação de citocinas e fatores de crescimento e a concomitante disfunção endotelial pode contribuir simultaneamente para ambos os processos de doença. Reforçando esta interação cardio-renal, Ferreira et al $^{6}$, demonstraram recentemente que a resolução do "status urêmico" com o transplante renal levou a uma melhora significativa da cardiomiopatia do paciente urêmico. 


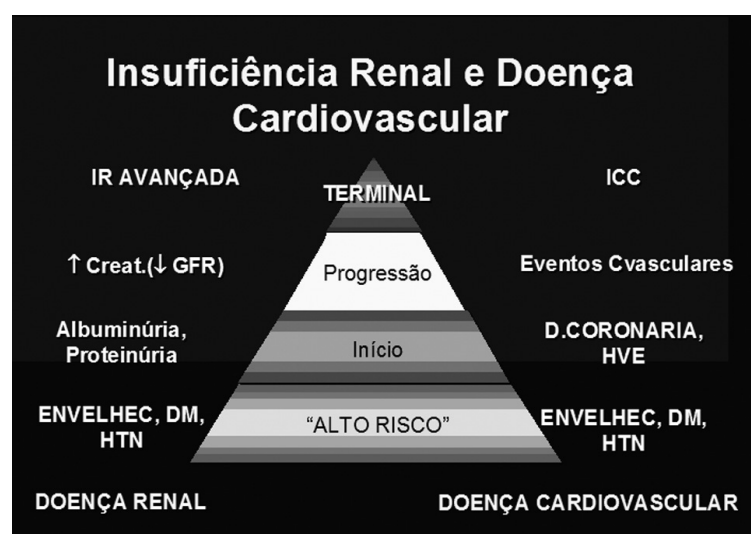

Figura 1. Evolução simultânea das doenças cardíaca e renal por influência dos fatores de risco.

Indivíduos saudáveis exibem um declínio fisiológico idade-dependente da função renal, com uma média de queda da taxa de filtração glomerular (TFG) após os 40 anos de idade de $0,75 \mathrm{ml} / \mathrm{min}$ por ano ${ }^{7}$. Uma vez instalado o dano renal, independente da etiologia, a redução anual da TFG pode ser acelerada e perpetuada pela presença de HAS, DM, proteinúria e dislipidemia ${ }^{8}$. Desde que na maioria dos casos a deterioração da função renal é assintomática, a detecção precoce e a intervenção são importantes para retardar a progressão da DRC.

Assim, um dos maiores objetivos do clínico, que se torna um grande desafio, é a prevenção da progressão da DRC. Como exemplo de intervenções utilizadas com este propósito estão: o controle da HAS, o uso de inibidores da enzima conversora de angiotensina (IECA) ou de bloqueadores do receptor da angiotensina II (BRA) e também o uso das estatinas para o controle do colesterol. Razões adicionais para o uso das estatinas na DRC incluem os possíveis benefícios no retardo da progressão da doença renal e na redução dos riscos de eventos cardiovasculares. Estes benefícios também podem estar relacionados aos efeitos secundários sobre a hemodinâmica renal, melhora da função endotelial, redução da proliferação das células mesangiais (e músculo liso vascular), efeito antiinflamatório e de estabilização de placa aterosclerótica ${ }^{9}$.

\section{Efeitos das estatinas na DRC}

\section{Complacência vascular e disfunção endotelial:}

A complacência vascular tem emergido como um dos preditores de mortalidade em pacientes em hemodiálise (HD) e após transplante renal ${ }^{10}$. As estatinas podem exercer efeitos benéficos sobre a complacência dos vasos e sobre a função endotelial na DRC.

Ichihara et al, ${ }^{11}$ examinaram a complacência de grandes artérias em indivíduos com DM tipo 2 em HD. Vinte e dois pacientes com níveis de lipídios normais receberam fluvastatina $(20 \mathrm{mg} / \mathrm{dia}$ ) ou placebo. Foram avaliados os níveis de lipídios, proteína C-reativa (PCR) e a complacência fluvastatina apresentou melhora na complacência arterial, nos níveis de LDL oxidado e de PCR.

Em pacientes com doença renal policística, um recente estudo ${ }^{12}$ mostrou que a sinvastatina levou a melhora na hemodinâmica renal, com aumento do fluxo plasmático renal e da TFG. Foi observada, ainda, melhora na vasodilatação induzida pela acetilcolina, mostrando melhora da função endotelial, provavelmente devido a aumento na produção de óxido nítrico.

\section{EFEITOS SOBRE A INFLAMAÇÃo NA IRC}

Processos inflamatórios são comuns em indivíduos com DRC, devido a diversos fatores como maior incidência de infecções, ambiente urêmico, diminuição da excreção de citocinas pró-inflamatórias e presença de aterosclerose difusa. Embora não haja definição precisa da prevalência de inflamação, há relatos de que 30 a $60 \%$ dos pacientes em diálise apresentam inflamação crônica ${ }^{13}$. A presença deste estado inflamatório correlaciona-se com a aterogênese acelerada, desnutrição e anemia.

Evidências apontam para ação antiinflamatória das estatinas em pacientes com insuficiência renal. Um estudo randomizado avaliou o efeito da sinvastatina (20 $\mathrm{mg} / \mathrm{dia}$ ) versus placebo sobre os marcadores de inflamação em 62 pacientes em hemodiálise com níveis de colesterol $>200 \mathrm{mg} / \mathrm{dl}$ (14). Após oito semanas de tratamento com sinvastatina, os pacientes apresentaram redução dos níveis de colesterol e de PCR e aumento nos níveis de albumina, alterações não vistas no grupo placebo.

\section{Efeitos das estatinas sobre a função renal}

Em animais, a dislipidemia é associada a dano glomerular, levando a glomeruloesclerose ${ }^{15,16}$. Entretanto, estudos em humanos não correlacionam certas condições de dislipidemia, como a hiperlipidemia familiar, com dano renal. Entretanto, uma vez que a DRC tenha sido instalada, algumas séries têm demonstrado uma associação entre progressão da DRC e hiperlipidemia ${ }^{17}$. Interessante que, em ratos espontaneamente hipertensos, as estatinas reduzem a proteinúria relacionada a HAS, de forma independente dos valores de pressão arterial e colesterol ${ }^{18}$.

O estudo CARE recrutou 4159 pacientes hiperlipidêmicos com história de infarto do miocárdio ${ }^{19}$. Foram randomizados para receber pravastatina ou placebo e seguidos por 5 anos. Sub-análise de 690 pacientes com TFG basal $<60 \mathrm{ml} / \mathrm{min}$ mostrou que, no grupo que recebeu a pravastatina, a redução da TFG com o tempo não foi diferente ao ser comparado com placebo. Entretanto, em um pequeno grupo de indivíduos com $\mathrm{TFG}<40 \mathrm{ml} / \mathrm{min}$, o tratamento com a pravastatina resultou em uma taxa de progressão da disfunção renal mais lenta que o grupo placebo. Os testes de interação sugerem que o benefício da pravastatina na prevenção 
da progressão da DRC foi mais pronunciado no grupo com proteinúria e pior função renal basal ${ }^{20}$.

O estudo GREACE ${ }^{21}$ avaliou 1600 pacientes com doença da artéria coronária estabelecida que foram alocados para receber atorvastatina ou placebo. Foi realizada sub-análise dos pacientes em relação à função renal utilizando valores de clearance de creatinina derivados da fórmula de Cockcroft-Gault. Após 36 meses de seguimento, os pacientes em uso de atorvastatina mostraram aumento de $12 \%$ na TFG, enquanto que os pacientes que não receberam estatinas apresentaram uma queda de $5,2 \%$ na TFG. Assim, a atorvastatina significativamente melhorou a função renal, efeito este provavelmente relacionado à melhora da vasodilatação mediada pelo endotélio ${ }^{22}$.

Outro estudo com atorvastatina avaliou a progressão da doença renal em 56 pacientes com doença renal préexistente já tratados com IECA / BRA que foram alocados para receber atorvastatina ou placebo. Após um ano, o tratamento com atorvastatina foi associado com redução na proteinúria (2,2 para 1,2 g/dia) e estabilização da função renal. No grupo placebo houve queda da TFG e nenhuma alteração na proteinúria ${ }^{23}$.

Fried et al. ${ }^{24}$ em uma meta-análise de 13 estudos envolvendo 404 pacientes, demonstraram que o uso de fármacos hipolipomiantes associou-se a um efeito protetor da progressão da disfunção renal.

Após o transplante renal, inúmeros fatores (imunológicos e não imunológicos) estão envolvidos na nefropatia crônica do enxerto, sendo bem mais complexo o estudo dos benefícios das estatinas neste contexto. Um recente estudo com a fluvastatina demonstrou redução no declínio da função do enxerto renal ${ }^{25}$. Em contrate, o ALERT 26 não demonstrou benefício da fluvastatina na redução da progressão da disfunção renal em pacientes transplantados renais. Porém em sub-análise deste estudo, em relação ao grupo placebo, o grupo fluvastatina apresentou significativas reduções do risco para morte cardíaca e infarto do miocárdio não fatal. Uma revisão recente de vários estudos sobre as estatinas em pacientes transplantados renais ${ }^{27}$, demonstrou benefícios destes fármacos sobre os marcadores de risco cardiovascular e possivelmente redução nos eventos cardiovasculares.

\section{SEgurança no uso de estatinas NA DRC}

A rabdomiólise é um efeito raro, porém grave, do uso de estatinas em pacientes com função renal normal (incidência $<1: 1000$ ). O risco de miopatia é maior quando a estatina é utilizada em altas doses e/ou quando associada a fibratos e ciclosporina. Quando estas associações são utilizadas, a pravastatina e a fluvastatina são os fármacos de escolha, devido a não interferência com a citocromo P450 (isoforma 3A4), levando a menor toxicidade muscular.
É importante ressaltar neste grupo de pacientes renais crônicos que existe a possibilidade de maior risco de lesão muscular, provavelmente em virtude do acúmulo das estatinas ou de seus metabólitos e pela injúria muscular relacionada à uremia. Entretanto, estudos realizados em pacientes com DRC em tratamento conservador ${ }^{19}$, em diálise ${ }^{28}$ e em transplantados renais ${ }^{26}$, sugerem um bom perfil de segurança.

A sinvastatina foi avaliada em 58 pacientes em programa de hemodiálise e diálise peritonial, em doses que variavam de 5 a $20 \mathrm{mg}$ por dia (média de $10 \mathrm{mg}$ por dia), apresentando bom perfil de segurança ${ }^{28}$. Em pacientes com proteinúria significativa ( $>1 \mathrm{~g} / \mathrm{dia}$ ), estudo duplo-cego controlado com placebo em 30 pacientes, mostrou que a sinvastatina, em doses que variaram de 10 a $40 \mathrm{mg} / \mathrm{dia}$, foi bem tolerada, não interferindo com a função renal e a proteinúria ${ }^{29}$. Da mesma forma, Olbricht ${ }^{30}$ mostrou a segurança da utilização da sinvastatina em paciente com síndrome nefrótica em estudo multi-cêntrico, duplo-cego, placebo-controlado, com a inclusão de 56 pacientes acompanhados por dois anos.

Em estudo duplo-cego placebo-controlado com 42 pacientes divididos em dois grupos quanto à função renal, a fluvastatina mostrou-se eficaz e segura em ambos os grupos, não apresentando maior incidência de efeitos adversos no grupo com insuficiência renal ${ }^{31}$.

Contudo, estimativa mais acurada de riscos com o uso de tais fármacos ainda não foi estabelecida em pacientes com DRC, seja em tratamento conservador ou em diálise, visto que os estudos excluíram tais pacientes ou foram realizados com populações pequenas.

\section{ESTATINAS E MORTALIDADE CARDIOVASCULAR NA DRC}

Diversos estudos na população geral demonstraram o benefício do tratamento com estatinas na redução da morbi-mortalidade cardiovascular. Não existem estudos controlados randomizados com amostras populacionais apropriadas que comprovem redução na incidência da DCV com o tratamento da dislipidemia em pacientes com DRC.

O papel da desnutrição e inflamação modulando o metabolismo lipídico dificulta traçar um paralelo entre o impacto do tratamento da dislipidemia e a redução da morbi-mortalidade cardiovascular nesta população. De fato, resultado da desnutrição e da reação inflamatória sistêmica, níveis reduzidos de colesterol observados nestes pacientes se associam com maior mortalidade cardiovascular.

Em concordância com as III Diretrizes Brasileiras Sobre Dislipidemias e Diretriz de Prevenção de Aterosclerose do Departamento de Aterosclerose da Sociedade Brasileira de Cardiologia ${ }^{32}$, a adequação dos valores de LDL-c é o alvo primário da terapêutica. Uma vez que os portadores de DRC são considerados pacientes de alto risco de eventos coronarianos, a meta de LDL-c deve ser $<100 \mathrm{mg} / \mathrm{dL}$. 
Não existem evidências de relação entre níveis aumentados de TG e morbi-mortalidade cardiovascular em portadores de DRC. No entanto, evidências extrapoladas da população geral permitem assumir esta provável associação. As estatinas proporcionam redução nos níveis de TG da ordem de 7 a $20 \%$ e provavelmente deve ser a escolha inicial para o tratamento desta dislipidemia na DRC, considerando o risco dos fibratos, embora não haja dados suficientes para definir a conduta ideal ${ }^{33}$.

Uma recente publicação envolvendo 1255 pacientes diabéticos em programa de hemodiálise comparou a utilização de atorvastatina $20 \mathrm{mg}$ com placebo durante 4 anos. Não se observou diferença significativa entre os grupos durante este período ${ }^{34}$.

Um estudo prospectivo em andamento (AURORA), randomizado, duplo-cego, placebo-controlado, terá como objetivo avaliar se a terapia com rosuvastatina poderá reduzir a mortalidade total e a incidência dos principais eventos cardiovasculares em 2700 pacientes com insuficiência renal em programa de hemodiálise.

Algumas questões com relação ao uso de estatinas em pacientes com DRC ainda permanecem sem resposta. Devemos utilizar o LDL-colesterol como alvo primário, ou escolher outros marcadores, como PCR e IL-6? Se o
LDL-colesterol for o alvo, qual o nível a ser atingido, visto que estes pacientes apresentam níveis reduzidos de colesterol devido à desnutrição?

As diretrizes da Sociedade Brasileira de Nefrologia ${ }^{33}$ estabelecem que:

- Pacientes portadores de DRC devem ser considerados como de alto risco para doença cardiovascular, ou seja, apresentam risco equivalente a indivíduos com insuficiência coronária conhecida.

* Nível de evidência: I

* Grau de recomendação: A

- Na ausência de dados específicos obtidos em estudos de populações de portadores de DRC, é razoável seguir as determinações do III NCEP para estabelecer metas e estratégias terapêuticas de correção das anormalidades lipídicas encontradas nos pacientes com DRC.

* Nível de evidência: III

* Grau de recomendação: B

- O tratamento das alterações lipídicas, principalmente através do uso de estatinas, em indivíduos com DRC pode retardar a evolução da doença renal.

* Nível de evidência: II

* Grau de recomendação: B

\section{REFERÊNCIAS}

1. Appel G. Lipid abnormalities in renal disease. Kidney Int 1991; 39: 169.

2. Mathur S, Devaraj S and Jialal I. Accelerated atherosclerosis, dyslipidemia, and oxidative stress in end-stage renal disease. Curr Opin Nephrol Hypertens 2002; 11: 141-147.

3. Sarnak MJ, Levey AS. Cardiovascular disease and chronic renal disease: A new paradigm. Am J Kidney Dis 2000; 35 (4 suppl 1): S117-S131.

4. Anavekar NS and Pfeffer MA. Cardiovascular risk in chronic kidney disease. Kidney Int 2004; 66 (s 92): S 11.

5. Levin A, Djurdjev O, Barrett B, Burgess E, Carlisle E, Ethier J, Jindal K, Mendelssohn D, Tobe S, Singer J, Thompson C. Cardiovascular disease in patients with chronic kidney disease: Getting to the heart of the matter. Am J Kidney Dis 2001; 38 (6): 1398-1407.

6. Ferreira SR, Moises VA, Tavares A, Pacheco-Silva A. Cardiovascular effects of successful renal transplantation: a 1-year sequential study of left ventricular morphology and function, and 24-hour blood pressure profile. Transplantation 2002; 74 (11): 1580-7.

7. Lindeman RD, Tobin J, Shock NW: Longitudinal studies on the rate of decline in renal function with age. J Am Geriatr Soc 1985; 33: 278.

8. Viberti GC, Bilous RW, Mackintosh D, Keen H. Monitoring glomerular fitration in diabetic nephropathy. Am J Med 1983; 74: 256.

9. Afzali B, Haydar AA, Vinen K, Goldsmith DJ. From Finland to fatland: beneficial effects of statins for patients with chronic kidney disease. J Am Soc Nephrol 2004; 15 (8): 2161-8.

10. Barenbrock M, Kosch M, Joster E, Kisters K, Rahn KH, Hausberg M. Reduced arterial distensibility is a predictor of cardiovascular disease in patients after renal transplantation. J Hypertens 2002; 20 (1): 79-84.

11. Ichihara A, Hayashi M, Ryuzaki M, Handa M, Furukawa T, Saruta T. Fluvastatin prevents development of arterial stiffness in haemodialysis patients with type 2 diabetes mellitus. Nephrol Dial Transplant 2002
12. van Dijk MA, Kamper AM, van Veen S, Souverijn JH, Blauw GJ. Effect of simvastatin on renal function in autosomal dominant polycystic kidney disease. Nephrol Dial Transplant 2001; 16 (11): 2152-7.

13. Yeun JY, Levine RA, Mantadilok V, Kaysen GA. C-Reactive protein predicts all-cause and cardiovascular mortality in hemodialysis patients. Am J Kidney Dis 2000; 35 (3): 469-76.

14. Chang JW, Yang WS, Min WK, Lee SK, Park JS, Kim SB: Effects of simvastatin on high-sensitivity $C$-reactive protein and serum albumin in hemodialysis patients. Am J Kidney Dis 2002; 39: 1213-1217.

15. Keane WF, Kasiske BL, O'Donnell MP. Lipids and progressive glomerulosclerosis. A model analogous to atherosclerosis. Am J Nephrol 1988; 8: 261

16. Lambert G, Sakai N, Vaisman BL, et al. Analysis of glomerulosclerosis and atherosclerosis in lecithin cholesterol acyltransferase-deficient mice. J Biol Chem 2001; 276: 15090.

17. Samuelsson O, Mulec H, Knight-Gibson C, et al. Lipoprotein abnormalities are associated with increased rate of progression of human chronic renal insufficiency. Nephrol Dial Transplant 1997; 12: 1908.

18. Yamashita T, Kawahsim S, Miwa Y, et al. A 3-hydroxy-3methylglutary co-enzyme A reductase inhibitor reduces hypertensive nephrosclerosis in stroke-prone spontaneously hypertensive rats. J Hyperten 2002 20: $2465-73$

19. Tonelli M, Moye L, Sacks FM, Kiberd B, Curhan G; Cholesterol and Recurrent Events (CARE) Trial Investigators. Pravastatin for secondary prevention of cardiovascular events in persons with mild chronic renal insufficiency. Ann Intern Med 2003; 138 (2): 98-104

20. Tonelli M, Moye L, Sacks FM, Cole T, Curhan GC. Effect of pravastatin on loss of renal function in people with moderate chronic renal insufficiency and cardiovascular disease. J Am Soc Nephrol 2003; 14: 1605.

21. Athyros VG, Papageorgiou AA, Mercouris BR, et al. Treatment with atorvastatin to the National Cholesterol Educational Program goa versus 'usual' care in secondary coronary heart disease prevention. 
The GREek Atorvastatin and Coronary-heart-disease Evaluation (GREACE) study. Curr Med Res Opin 2002; 18: 220.

22. Athyros VG, Mikhailidis DP, Papageorgiou AA, et al. The effect of statins versus untreated dyslipidaemia on renal function in patients with coronary heart disease. Journal of Clinical Pathology. In Press 2004.

23. Bianchi S, Bigazzi R, Caiazza A, Campese VM. A controlled, prospective study of the effects of atorvastatin on proteinuria and progression of kidney disease. Am J Kidney Dis 2003; 41: 565.

24. Fried LF, Orchard TJ, Kasiske BL. Effect of lipid reduction on the progression of renal disease: a meta-analysis. Kidney Int 2001; 59: 260.

25. Konstadinidou I, Boletis JN, Perrea D, Triantafyllou A, Filiopoulos V, Stamataki E, Kostakis A, Stathakis C: Beneficial effects of fluvastatin on progreeive renal allograft dysfunction. Transplant Proc 2003; 35 : 1364-1367.

26. Holdaas H, Fellstrom B, Jardine AG, Holme I, Nyberg G, Fauchald P, Gronhagen-Riska C, Madsen S, Neumayer HH, Cole E, Maes B, Ambuhl P, Olsson AG, Hartmann A, Solbu DO, Pedersen TR; Assessment of LEscol in Renal Transplantation (ALERT) Study Investigators. Effect of fluvastatin on cardiac outcomes in renal transplant recipients: a multicentre, randomised, placebo-controlled trial. Lancet2003;361(9374): 2024-31.

27. Lentine KL, Brennan DC. Statin use after renal transplantation: a systematic quality review of trial-based evidence. Nephrol Dial Transplant 2004; 19 (9): 2378-86.
28. Saltissi D, Morgan C, Rigby RJ, Westhuyzen J. Safety and efficacy of simvastatin in hypercholesterolemic patients undergoing chronic renal dialysis. Am J Kidney Dis 2002; 39 (2): 283-90.

29. Thomas ME, Harris KP, Ramaswamy C, Hattersley JM, Wheeler DC Varghese Z, Williams JD, Walls J, Moorhead JF. Simvastatin therapy for hypercholesterolemic patients with nephrotic syndrome or significant proteinuria. Kidney Int 1993; 44 (5): 1124-9.

30. Olbricht CJ, Wanner C, Thiery J, Basten A, for the Simvastatin In Nephrotic Syndrome Study Group. Simvastatin In Nephrotic Syndrome. Kidney Int 1999; 56 (Suppl 71): S113-S116.

31. Lintott CJ, Scott RS, Bremer JM, Shand BI. Fluvastatin for dyslipoproteinemia, with or without concomitant chronic renal insufficiency. Am J Cardiol 1995; 76 (2): 97A-101A.

32. III Diretrizes Brasileiras Sobre Dislipidemias e Diretriz de Prevenção de Aterosclerose do Departamento de Aterosclerose da Sociedade Brasileira de Cardiologia. Arq Bras Cardiol 2001; 77 (Supl III): 1-48

33. Diretrizes Brasileiras de Doença Renal Crônica. J Bras Nefrol 2004 XXVI (3) Supl 1

34. Warner $\mathrm{C}$ et al. Atorvastatin in patients with type 2 diabetes mellitus undergoing hemodialysis. N Engl J Méd. 2005;353:238-48. 